\title{
Induction and transport of Wnt 5a during macrophage-induced malignant invasion is mediated by two types of extracellular vesicles
}

\author{
Kerstin Menck ${ }^{1, *}$, Florian Klemm ${ }^{1, *}$, Julia Christina Gross ${ }^{2}$, Tobias Pukrop ${ }^{1}$, Dirk \\ Wenzel $^{3}$, Claudia Binder ${ }^{1}$ \\ ${ }^{1}$ Dept of Hematology/Oncology, University Medicine, Göttingen, Germany \\ 2 Div. Signaling and Functional Genomics, German Cancer Research Center, Heidelberg, Germany \\ ${ }^{3}$ Max Planck Institute for Biophysical Chemistry, Göttingen, Germany \\ ${ }^{*}$ These authors contributed equally to this work.
}

Correspondence to: Claudia Binder, email: cbinder@med.uni-goettingen.de

Keywords: Breast cancer, exosomes, Evi, microvesicles, macrophages, Wnt 5 a

Received: August 27, $2013 \quad$ Accepted: October 19, $2013 \quad$ Published: October 21, 2013

This is an open-access article distributed under the terms of the Creative Commons Attribution License, which permits unrestricted use, distribution, and reproduction in any medium, provided the original author and source are credited.

\section{ABSTRACT:}

Recently, we have shown that macrophage (MФ)-induced invasion of breast cancer cells requires upregulation of $\mathrm{Wnt} 5 \mathrm{a}$ in $\mathrm{M \Phi}$ leading to activation of $\beta$-Cateninindependent Wnt signaling in the tumor cells. However, it remained unclear, how malignant cells induce Wnt 5 a in $M \Phi$ and how it is transferred back to the cancer cells. Here we identify two types of extracellular particles as essential for this intercellular interaction in both directions. Plasma membrane-derived microvesicles (MV) as well as exosomes from breast cancer cells, although biologically distinct populations, both induce Wnt 5a in MФ. In contrast, the particle-free supernatant and vesicles from benign cells, such as platelets, have no such effect. Induction is antagonized by the Wnt inhibitor Dickkopf-1. Subsequently, Wnt 5a is shuttled via responding MФ-MV and exosomes to the tumor cells enhancing their invasion. Wnt 5a export on both vesicle fractions depends at least partially on the cargo protein Evenness interrupted (Evi). Its knockdown leads to Wnt 5a depletion of both particle populations and reduced vesicle-mediated invasion. In conclusion, MV and exosomes are critical for MФ-induced invasion of cancer cells since they are responsible for upregulation of MФWnt 5 a as well as for its delivery to the recipient cells via a reciprocal loop. Although of different biogenesis, both populations share common features regarding function and Evi-dependent secretion of non-canonical Wnts.

\section{INTRODUCTION}

Infiltrating macrophages $(\mathrm{M} \Phi)$ are involved in the various steps of tumor progression from primary cancer cell invasion to metastatic colonization of distant tissues [1]. They are characterized by a tumor-promoting M2profile of secreted cytokines which differs from that of classically activated M1-M $\Phi$ by high abundance of IL10 and -4 and low amounts of IL- $1 \beta$ [2]. TNF $\alpha$ is also critical for the proinvasive effect of $\mathrm{M} \Phi$ [3] governing the induction of matrix metalloproteinases (MMP) which are necessary for extracellular matrix break-down during invasion [4].
How cancer cells communicate with infiltrating $\mathrm{M} \Phi$ to elicit a tumor-promoting phenotype is still not completely clear. Several soluble factors have been identified, such as CSF-1, which engages in a reciprocal proinvasive loop with MФ-derived EGF [5]. We have shown that MФ-induced invasion of breast cancer cells requires upregulation of Wnt $5 \mathrm{a}$ in $\mathrm{M} \Phi$ and activation of $\beta$-Catenin-independent Wnt signaling in the tumor cells [6]. Wnt ligands, especially when signaling through the canonical Wnt/ $\beta$-Catenin pathway, play a critical role in proliferation and migration during embryonic development [7] as well as in malignant transformation and progression. The $\beta$-Catenin-independent ligand Wnt 5 a can act either as 
a tumor promoter [8] or suppressor in a context-dependent way [9]. It is expressed not only in tumor cells, where it regulates MMP transcription and migration, but also during inflammatory reactions of $\mathrm{M} \Phi[10]$. The secretion of Wnts requires lipid modification by the acyltransferase porcupine in the endoplasmatic reticulum from where they are shuttled to the Golgi apparatus and transported to the plasma membrane by binding to the seven passtransmembrane protein Eveness interrupted (Evi) [11]. Distribution of the hydrophobic Wnts outside the cell is still a matter of debate and can occur, among other ways, by attachment to extracellular membranous vesicles [12].

Mammalian cells produce these particles both constitutively as well as following various triggers [13]. Composition, size and function differ according to their biogenesis. Three main populations can be distinguished: exosomes ((Exo) approx. 50 - $100 \mathrm{~nm})$, microvesicles ((MV) $100-1000 \mathrm{~nm})$ and apoptotic bodies $(1-5 \mu \mathrm{m})$ [14]. The latter are products of dying cells and contain intracellular organelles or fragments of these. Exo are derived from endosomal vesicles and released via formation of multivesicular bodies (MVB) and fusion with the plasma membrane. In contrast, MV directly bud off from the outer plasma membrane. MV often expose phosphatidyl serine (PS) and tissue factor which explains their procoagulatory function [15], as well as various surface molecules of the respective parent cell [16]. They also contain hydrophilic proteins, frequently enriched in comparison to the parent cell, such as IL$1 \beta$, for which they provide a mode of atypic secretion [17]. A similar function has been reported for Exo which export biologically active Drosophila Wingless as well as mammalian Wnt 3a in an Evi-dependent way [18]. In neurons, the Evi-Wnt complex can be transferred via exosomal vesicles across synapses, indicating a trafficking function of Evi not only in the Wnt-secreting but also in the accepting cells [19]. An association of Wnt-Evi with $\mathrm{MV}$, particularly in the human system, has not yet been described.

While there is increasing evidence that Exo play a role in Wnt secretion and also in cancer development [20], much less is known on MV in this context. Tumor cell-derived MV have been found in various body fluids of cancer patients [21], [22], their amount correlating with advanced stage. Although their effect has originally been attributed to their prothrombotic activity, recent data indicate a direct involvement in cancer progression. MV induce transformation via horizontal transfer of the mutated EGF receptor [23] and interfere with immune reactions by transmission of MHC molecules [24] and interaction with PS receptors [25]. They modulate the activity of MФ via transport of HSP 70 [26], induction of IL-10 as part of an anti-inflammatory phenotype [27] and by shuttling of IL-8 and VEGF mRNA [28]. In a murine model of renal carcinoma, MV trigger angiogenesis and promote metastasis [29]. Malignant invasion is enhanced via reciprocal exchange of MV between prostate cancer cells and fibroblasts [30] as well as by MV-induced MMP upregulation [31].

Based on these findings we hypothesized that extracellular particles are attractive candidates for the intercellular communication between cancer cells and infiltrating $М \Phi$. We therefore investigated the role of MV and exosomes from either cell type in MФ-induced invasion of breast cancer cells and in the regulation and transport of $\mathrm{M} \Phi-W n t 5 a$ which is crucial for this process.

\section{RESULTS}

\section{Microvesicles and exosomes are distinct vesicle populations}

In a coculture system without direct cell-cell contact, we have previously shown that cancer cells induce upregulation of Wnt 5a in $M \Phi$ which then leads to enhanced malignant invasion. We now aimed to clarify whether this is mediated by soluble or vesicle-associated components of the supernatant. MV and Exo from breast cancer cell lines (T-MV/-Exo), peripheral blood-derived $\mathrm{M} \Phi$ (MФ-MV/-Exo) and donor platelets (P-MV/-Exo) as a control were prepared by differential centrifugation without any previous stimulation. Particular care was taken to obtain non-activated P-MV/-Exo populations by isolating the vesicles directly from the concentrates without any additional stimulation step as used by many other authors. Analysis of the MV fraction by transmission electron microscopy (TEM) yielded membrane-enclosed particles with the typical irregular shape of MV and a diameter between 100 and $1000 \mathrm{~nm}$. The Exo fraction, in contrast, contained smaller, often cup-shaped vesicles (Fig. 1 A, B). There was no evidence of apoptotic bodies, which, according to their size, would appear in the MV fraction. Membrane integrity and the vital nature of the MV were confirmed by staining with calcein AM, fluorescent only after enzymatic metabolization (Fig. $1 \mathrm{C}$ ). The tumor vesicles were further characterized regarding the expression of typical proteins (Fig. 1 D - F). CD63 and the cytosolic tumor susceptibility gene TSG101, both considered specific for Exo, were present on T-Exo, but not on T-MV from SK-BR-3 cells. As a marker for T-MV, we identified the plasma membrane-bound extracellular MMP inducer EMMPRIN, which was present exclusively on T-MV. The same results were achieved with MCF-7 vesicles (not shown). Together this indicates that the centrifugation protocol effectively separated the two populations and that they are in fact biologically distinct. 


\section{Tumor cell-derived vesicles induce production of Wnt 5a-positive MФ vesicles which then induce invasion}

We next asked whether these particles are responsible for the upregulation of Wnt $5 \mathrm{a}$ in $M \Phi$ which is the critical step during $\mathrm{M} \Phi$-induced invasion. Therefore, $M \Phi$ were exposed to T-MV and T-Exo from MCF-7 cells. Both vesicles induced Wnt 5a mRNA in MФ in a concentration-dependent way while the respective supernatant and P-MV as a benign control did not (Fig. 2 A). MCF-7 cells constitutively express very low amounts of Wnt 5a mRNA raising the possibility that the increase in $M \Phi-W n t ~ 5 a$ was due to horizontal mRNA transfer. We therefore exposed M $\Phi$ to T-MV with concomitant inhibition of mRNA transcription by actinomycin D (ActD) (Fig. 2 B) in concentrations not interfering with $\mathrm{M} \Phi$ viability. Under these conditions, T-MV did not increase Wnt 5a mRNA, indicating that Wnt $5 \mathrm{a}$ in $\mathrm{M} \Phi$ is newly transcribed. Wnt 5 a induction could be confirmed on the protein level. In contrast to unstimulated $\mathrm{M} \Phi$, Wnt 5 a was increasingly detectable not only in whole MФ exposed to T-MV but also in the respective MV and Exo (Fig. 2 C). As demonstrated by TEM and immunogold staining, Wnt 5a localized to the outer membrane of these MV (Fig. 2 D). Together, this indicates that Wnt 5a export
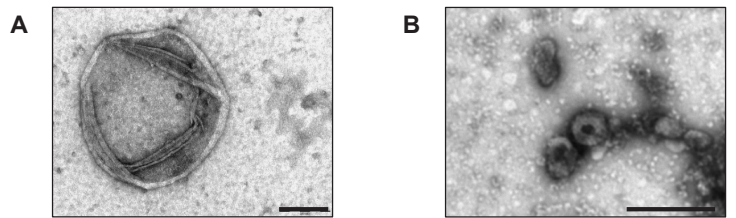

C

D
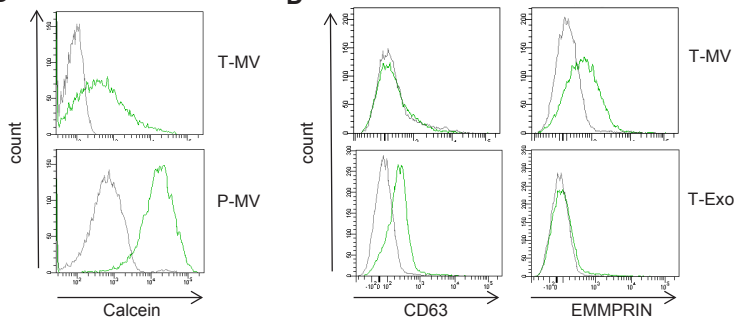

E

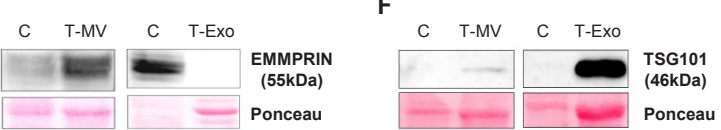

Figure 1: MV and Exo are distinct vesicle populations. (A) TEM of MV released from unstimulated SK-BR3 cells (TMV) showing spherical membrane vesicles with a diameter of $>100 \mathrm{~nm}$ (scale bar $=200 \mathrm{~nm}$ ). (B) TEM of Exo from the same cells $($ scale bar $=200 \mathrm{~nm}) .(\mathrm{C}+\mathrm{D})$ Flow cytometry $($ gray $=$ isotype control, green $=$ stained cells): (C) Calcein staining of T-MV and platelet P-MV confirming the membrane integrity. (D) Analysis of CD63 and EMMPRIN on SK-BR3 vesicles. $(\mathrm{E}+\mathrm{F})$ Western blot of EMMPRIN and TSG101 in whole cell lysates $(=\mathrm{C})$ and vesicles (blot exposure time 4-fold longer for $\mathrm{C} /$ Exo than for $\mathrm{C} / \mathrm{MV}$ ). from $М \Phi$ occurs at least partly via extracellular vesicles.

We then analyzed the individual effects on invasion of both kinds of vesicles and the particle-free supernatant derived from $\mathrm{M} \Phi$ pre-stimulated with T-MV as described above. In Boyden chamber microinvasion assays MCF7 cells responded with significantly enhanced invasion to addition of these MФ-MV and Exo (Fig. 2 E), but not to incubation with the respective particle-free supernatant. Equally, P-MV as well as their supernatant had no effect.

Wnt 5a export on MV and Exo could be identified also in other cell types. Wnt 5a was strongly enriched in MV and Exo from the constitutively Wnt 5a-positive cell line SK-BR-3 (Fig. 3 A). It was absent in MCF-7, platelets, unstimulated $M \Phi$ and the respective MV. In a sucrose gradient performed separately for the MV as well as the Exo fraction of SK-BR-3 cells, Wnt 5a colocalized partly with EMMRPIN and partly with TSG 101, underlining that it is present on both vesicle populations (Fig. 3 B).
A

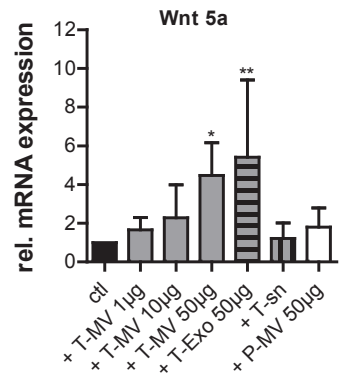

C

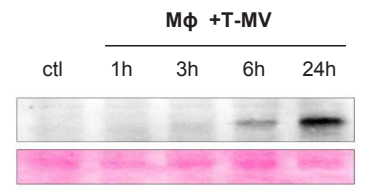

D

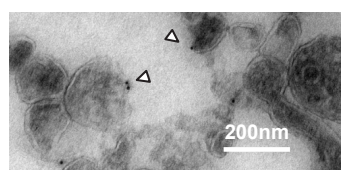

B

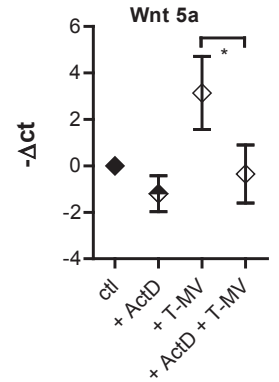

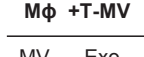

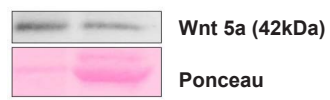

E

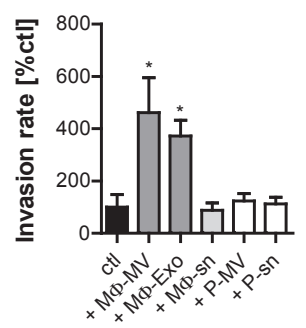

Figure 2: Tumor cell-derived vesicles induce production of Wnt 5a-positive MФ vesicles which then induce invasion. (A) $W N T 5 \mathrm{~A}$ mRNA is upregulated after 24h in MФ by T-MV from MCF-7 cells but neither by P-MV nor by the tumor cell supernatant (sn) (qRT-PCR, mean \pm SD, $\left.\mathrm{n}=5, \quad{ }^{*} \mathrm{p}<0,001, \quad * * \mathrm{p}<0.01, \quad * * * \mathrm{p}<0.05\right)$. (B) Inhibition of mRNA synthesis in $\mathrm{M} \Phi$ by ActD $(32 \mu \mathrm{M})$ antagonizes $W N T 5 A$ upregulation by T-MV (qRT-PCR, $n=3, p<0.05$ ). (C) Western blot: Upregulation of Wnt $5 \mathrm{a}$ in $\mathrm{M} \Phi$ by $50 \mu \mathrm{g}$ T-MV as well as in MФ-MV and Exo, isolated after $24 \mathrm{~h}$. (D) Immunogold staining of Wnt 5a on MV from T-MV-stimulated MФ (TEM). (E) Microinvasion assay. Exposure of MCF-7 cells to $1 \mu \mathrm{g}$ of particles as indicated induces invasion, while the particle-free supernatant (sn) and P-MV do not (means $\pm \mathrm{SD}, \mathrm{n}=3,{ }^{*} \mathrm{p}<0,001$ ). 


\section{Incorporation of tumor vesicles into $М \Phi$ is not required for Wnt 5a induction}

We then investigated whether uptake of the tumor vesicles, in particular T-MV, by $\mathrm{M} \Phi$ is a

A

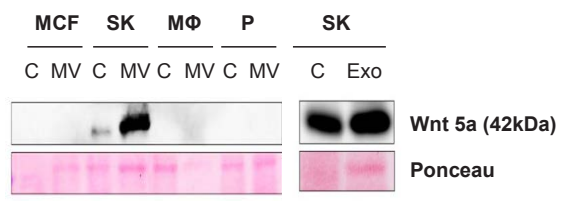

B
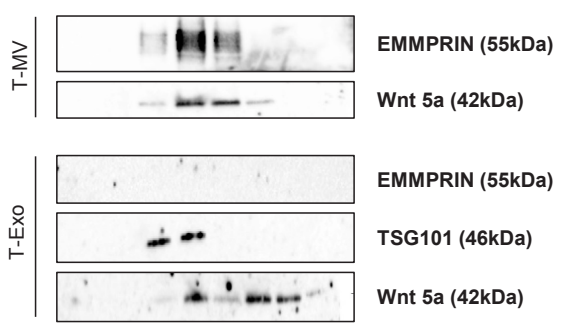

EMMPRIN (55kDa)

TSG101 (46kDa)

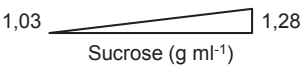

Wnt 5a (42kDa)

Figure 3: Wnt 5a is also exported on MV and Exo of SK-BR-3 cells. (A) Western blot: Wnt $5 \mathrm{a}$ is enriched in MV and Exo from SK-BR-3, but absent in MV from MCF-7, unstimulated $M \Phi$ and platelets. (B) Sucrose gradient of the T-MV and the T-Exo fraction from SK-BR-3, showing partial colocalization of Wnt 5a with the MV marker EMMPRIN and the exosomal marker TSG 101.
A

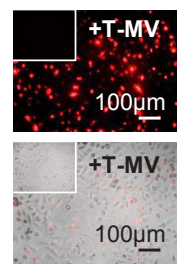

E

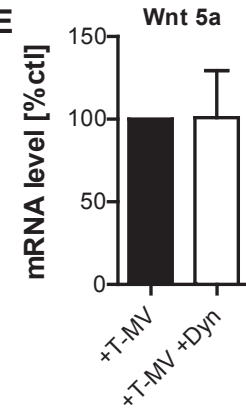

B

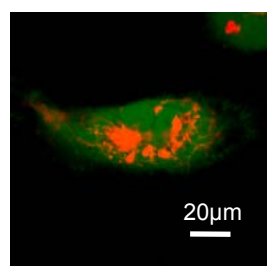

prerequisite for Wnt 5a induction. After 24 hours, a considerable amount of PKH26-stained T-MV could be identified inside of the MФ with a respective decrease of fluorescence in the supernatant (Fig. 4 A-C). However, pre-incubation with the endocytosis inhibitor dynasore in concentrations which did not interfere with cell viability (suppl. Fig. $1 \mathrm{~A}$ ), but effectively inhibited MV incorporation, did not abolish Wnt 5 a induction in $\mathrm{M} \Phi$ (Fig. $4 \mathrm{D}, \mathrm{E}$ ). This suggests that the contact between vesicles and the $M \Phi$ plasma membrane is sufficient to trigger Wnt 5a production. Dynasore alone had no effect on Wnt 5a expression (suppl Fig. 1 B). On the other side of the vesicle-mediated communication, also cancer cells exposed to Wnt 5a-positive $\mathrm{M} \Phi-\mathrm{MV}$ rapidly ingested these particles (Fig. 4 F). To trace the MV-delivered Wnt $5 \mathrm{a}$ protein in the receiving cells, we used MV from Wnt 5a-overexpressing L cells (Wnt 5a-L cells). MV-associated Wnt 5a was detectable in recipient MCF-7 cells, as were high doses of recombinant human Wnt 5 a used as control (Fig. 4 G). There was no influence of either rhWnt $5 \mathrm{a}$ or MV from wild type and Wnt 5a-L cells on Wnt 5a mRNA transcription (Fig. $4 \mathrm{H}$ ).

To clarify whether T-MV regulate additional secreted factors and induce a shift to a tumor-promoting M2-profile, selected cytokines were measured in MФ and the supernatant. Expression of the typical M1-markers IL$1 \beta$ and TNF $\alpha$ as well as of the M2-markers VEGF and IL-
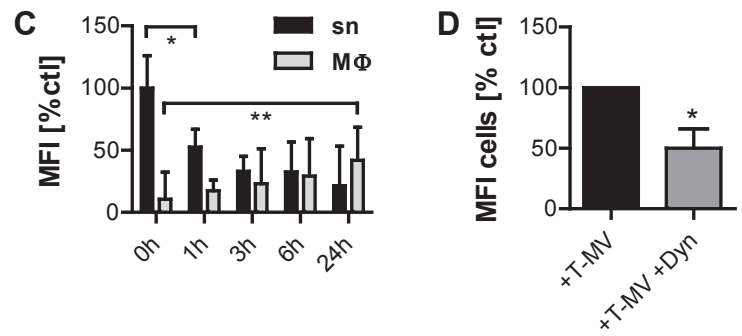

F

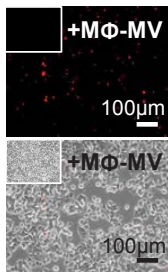

Wnt5a (42kDa)

Ponceau
G

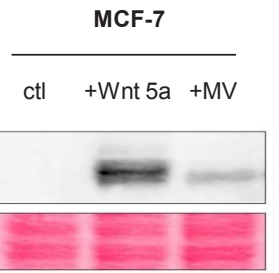

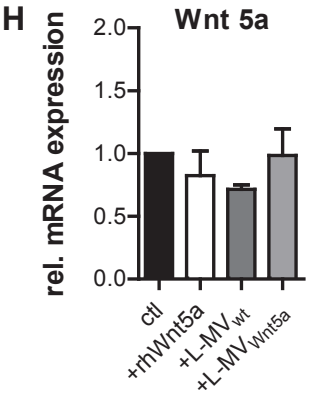

Figure 4: MV are mutually incorporated by $\mathbf{M \Phi}$ and tumor cells. (A-C) Uptake (24h) of PKH26-labeled T-MV from MCF7 into MФ: (A) fluorescence/bright field $(10 \times$, inserts = controls), (B) confocal microscopy (40×, green = calcein). (C) Quantification of PKH fluorescence in M $\Phi$ and sn by flow cytometry (means $\pm \mathrm{SD}, \mathrm{n}=5, * \mathrm{p}<0,01,{ }^{*} \mathrm{p}<0,05$ ). (D) Preincubation (2h) with dynasore $(12,5 \mu \mathrm{M})$ inhibits PKH26-labeled T-MV uptake (flow cytometry, $\mathrm{n}=3$, mean $\pm \mathrm{SD},{ }^{*} \mathrm{p}<0,05$ ), but does not counteract T-MV-induced

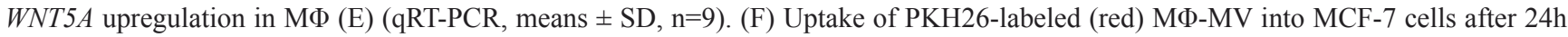
(fluorescence/bright field microscopy, 10x, inserts = controls). (G) Western blot: Wnt 5a delivered by MV from Wnt 5a-L cells as well as recombinant human Wnt $5 \mathrm{a}(100 \mathrm{ng} / \mathrm{ml})$ are detectable in whole MCF-7 lysates. (H) No induction of $W N T 5 A$ mRNA in MCF-7 by rhWnt 5 and MV from wt as well as Wnt 5a-L cells (qRT-PCR, means \pm SD, n=9). 
10 did not differ significantly upon exposure to increasing amounts of T-MV. Addition of non-proinvasive P-MV led to a significant increase of IL-1ß mRNA, suggesting a potential shift to an M1-phenotype with anti-tumor functions. However, this could not be confirmed on the protein level in MФ-supernatants (Fig. 5 A, B). Moreover, there was no effect of T-MV on the mRNA concentrations of various MФ-derived MMPs (Fig. 5 C).

\section{Dickkopf-1(DKK-1) antagonizes Wnt 5a induction in $\mathrm{M \Phi}$}

Earlier, we had identified the Wnt-inhibitor DKK-1 as an antagonist of the complete process of $\mathrm{M} \Phi$-induced invasion. To specify its point of action, we separately analyzed its influence on T-MV-mediated Wnt 5a transcription in $\mathrm{M} \Phi$ as well as on invasion induced by Wnt 5a-positive MФ-MV. The first step was effectively antagonized by DKK-1 (Fig. 6 A). However, the proinvasive effect of Wnt 5a-positive MФ-MV was not inhibited by DKK-1, in contrast to invasion induced by coculture with whole MФ (Fig. 6 B). This suggests that DKK-1 does not so much interfere with the action of Wnt $5 \mathrm{a}$ on the recipient tumor cells, but with its production in МФ. As a downstream event, p38/MAPK was rapidly phosphorylated upon incubation of MФ with T-MV from MCF-7 cells (Fig. 6 C). This was counteracted by DKK-1 (Fig. 6 D). Underlining the impact of $\mathrm{p} 38$ phosphorylation on T-MV-mediated induction of M $\Phi-W n t 5 a$, application of the p38/MAPK inhibitor SB-203580 significantly antagonized this process (Fig. $6 \mathrm{E}$ ).
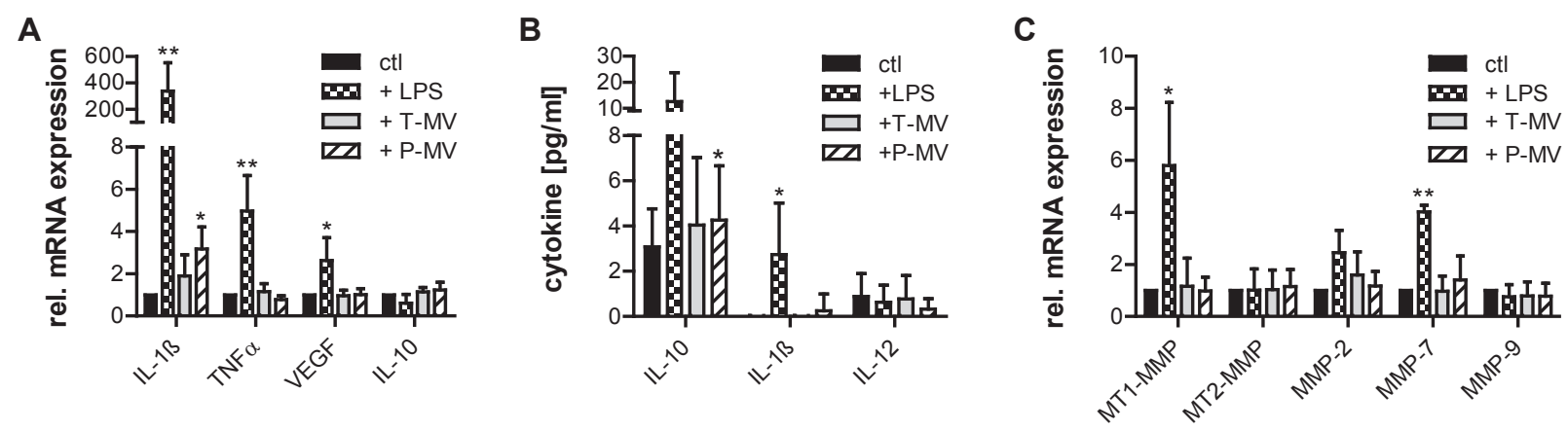

Figure 5: Tumor vesicles do not induce typical M2 features in MФ. (A) qRT-PCR for selected cytokines in MФ after stimulation with LPS $(100 \mathrm{ng} / \mathrm{ml})$, T-MV $(25 \mu \mathrm{g} / \mathrm{ml})$ and P-MV $(25 \mu \mathrm{g} / \mathrm{ml})$ for $24 \mathrm{~h}\left(\right.$ mean $\left.\pm \mathrm{SD}, \mathrm{n}=5,{ }^{*} \mathrm{p}<0,05\right)$. (B) Cytokine release by MФ (conditions see above, means $\left.\pm \mathrm{SD}, \mathrm{n}=5,{ }^{*} \mathrm{p}<0,05\right)$. (C) mRNA expression of selected MMPs by $\mathrm{M} \Phi$ stimulated as described above (qRT-PCR, means $\pm \mathrm{SD}, \mathrm{n}=3, * \mathrm{p}<0,05)$.

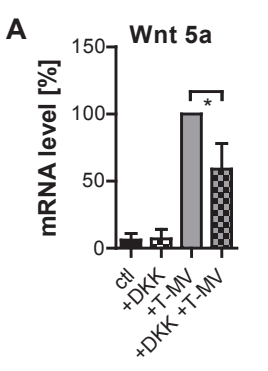

D

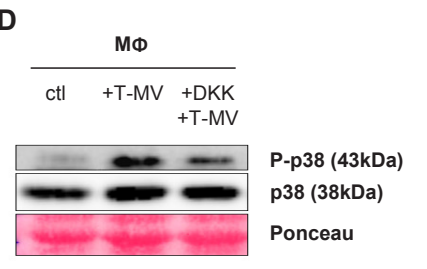

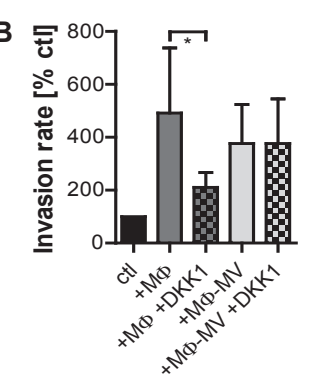

C
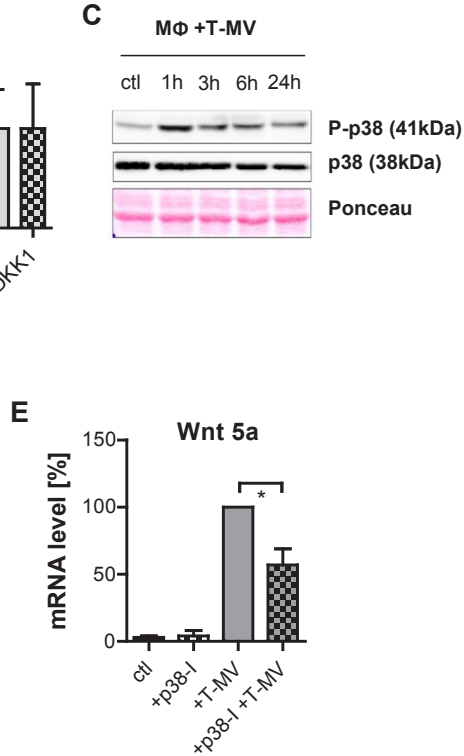

Figure 6: Vesicle-induced Wnt 5a-induction is Wnt- and p38-dependent. (A) DKK-1 antagonizes T-MV-mediated (50 $\mu$ g)

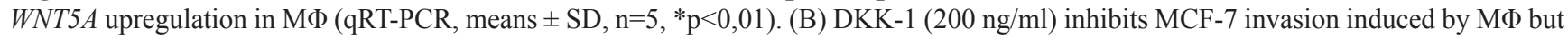
not by MФ-MV (microinvasion assay, means $\pm \mathrm{SD}, \mathrm{n}=3,{ }^{*} \mathrm{p}<0.001$ ). (C) Phosphorylation of $\mathrm{p} 38$ (Western blot) in $\mathrm{M} \Phi+\mathrm{T}-\mathrm{MV}$, which is antagonized by DKK-1 (D). (E) WNT5A induction is counteracted by SB-203580 (SB) (qRT-PCR, means \pm SD, $\mathrm{n}=3,{ }^{*} \mathrm{p}<0.05$ ). 


\section{Wnt 5a associates with vesicles in a non-specific as well as a specific way involving Evi}

To investigate whether Wnt 5a non-specifically associates with vesicle membranes, Wnt 5a-negative tumor vesicles were exposed to high concentrations of rhWnt5a. After $24 \mathrm{~h}$, a considerable part of the recombinant protein was detectable on MV and Exo, while a significant amount still remained in the supernatant (Fig. 7 A). However, apart from this non-specific attachment, we could also identify a specific component of the vesicle-associated Wnt 5a export. This involves the cargo protein Evi, recently described as essential for the secretion of Wnt

A
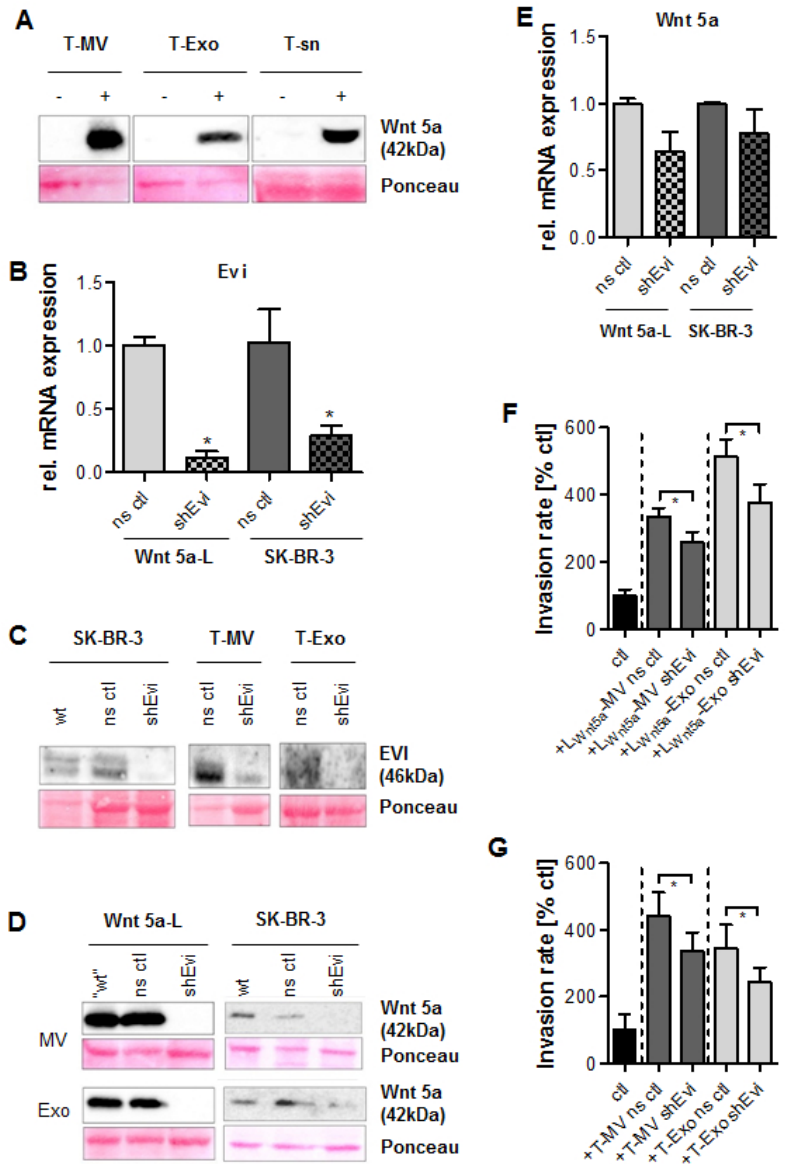

G

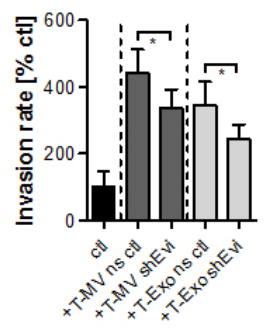

Figure 7: Evi knockdown leads to Wnt 5a depletion on both types of vesicles and inhibits invasion. (A) Western Blot: rhWnt $5 \mathrm{a}(100 \mathrm{ng} / \mathrm{ml}, 24 \mathrm{~h})$ to some extent associates with MCF-7 vesicles, but also remains in the particle-free supernatant (sn). (B) Stable knockdown of WLS/EVI mRNA by shEvi in Wnt 5a-L and SK-BR-3 cells (qRT-PCR, means \pm SD, $n=3,{ }^{*} \mathrm{p}<0.05$, ns $\mathrm{ctl}=$ nonsense control). (C) Western blot: Downregulation of Evi protein in SK-BR-3 cells and their vesicles by shEvi. (D) Western blot: Wnt 5a depletion in MV and Exo from Evi knockdown cells. (E) Influence of shEvi on WNT5A mRNA expression (qRT-PCR, means $\left.\pm \mathrm{SD}, \mathrm{n}=3,{ }^{*} \mathrm{p}<0.05\right)$. (F, G) Microinvasion assays: Evi knockdown antagonizes the increase of MCF-7 invasion induced by MV and Exo from (F) Wnt5a-L and (G) SK-BR-3 cells (means \pm SD, $n=3,{ }^{*} p<0.001$ ). 3a on exosomes by a member of our group. We used Wnt $5 \mathrm{a}-\mathrm{L}$ cells as well as the breast cancer cell line SK-BR-3 as models for Wnt 5a-positive MФ. Stable downregulation of Evi expression in these cell lines was performed by short hairpin RNA-mediated silencing (shEvi) (Fig. 7 B). Evi knockdown led to almost complete absence of the Evi protein in SK-BR-3 cells and their vesicles (Fig. 7 C). This was accompanied by downregulation of Wnt $5 \mathrm{a}$ not only on Exo but also on MV from both cell types (Fig. 7 D). This seems surprising since plasma membrane-derived MV - as we have shown before - are distinct from Exo and differ from these in their biogenesis and their cargo.

The shEvi effect on Wnt 5a was restricted to the protein since there was no significant reduction of Wnt $5 \mathrm{a}$ mRNA in both SK-BR-3 and Wnt 5a-L cells (Fig. 7 E). On the functional level, Wnt 5a depletion of MV and Exo from either cell line was followed by a significant decrease of vesicle-induced invasion of MCF-7 cells (Fig. 7 F, G), indicating that vesicle-associated Wnt $5 \mathrm{a}$ is essentially involved in MФ-induced invasion.

\section{DISCUSSION}

Previously, we have shown that $\mathrm{M} \Phi$-induced cancer cell invasion depends on Wnt 5a induction in $M \Phi$ as an essential part of their proinvasive phenotype. Now we demonstrate that this is not mediated by cancer cellderived soluble factors but by extracellular vesicles. MV and Exo from benign cells do not induce Wnt $5 \mathrm{a}$ in МФ.

Surprisingly, we identified Wnt 5a, induced by T-MV and T-Exo, not only in whole $\mathrm{M} \Phi$ but also in $M \Phi-M V$ and Exo which are produced in response. Equally, Wnt 5a-L cells and the Wnt 5a-positive breast cancer cell line SKBR-3 showed enrichment of Wnt $5 \mathrm{a}$ in their vesicles. The TEM images of MФ-MV suggest that Wnt $5 \mathrm{a}$ is located at the MV membrane. Wnts are able to non-selectively

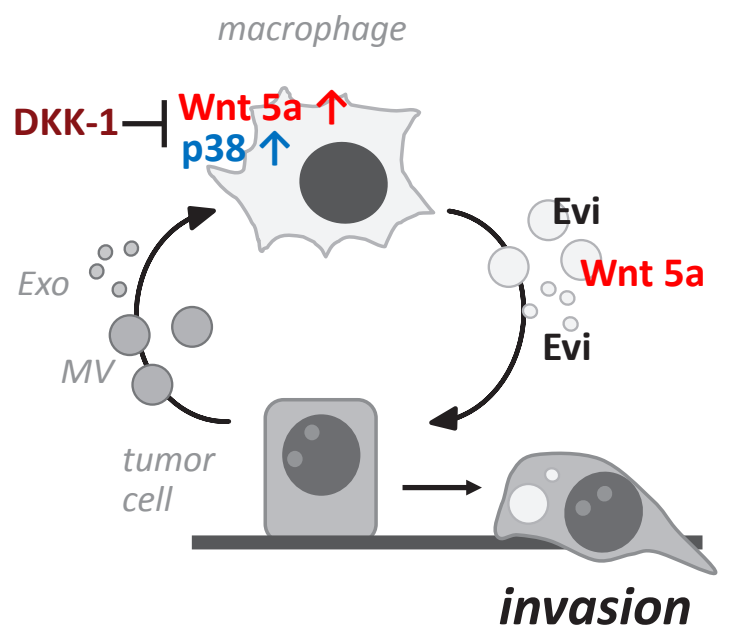

Figure 8: Schematic presentation of the vesiclemediated reciprocal loop during macrophage-induced tumor cell invasion 
"stick" to membranes due to their lipid modifications [12]. Consistently, we show that Wnt 5a-negative tumor vesicles can be loaded with recombinant Wnt $5 \mathrm{a}$, part of which remains soluble in the supernatant. Thus, Wnt 5a may indeed non-specifically associate with $M \Phi$ vesicles after secretion by stimulated MФ. However, our experimental evidence suggests that there is also a specific route of Wnt 5 a excretion through extracellular vesicles involving the protein Evi. This protein present on Exo and MVB has recently been reported to provide an alternative way of Wnt 3a secretion [18]. Until now, a role of Evi in the secretion of non-canonical Wnts as well as an association with MV has not been described. Here, we show that Evi is expressed not only on Exo but also on MV, a particle population considered biologically different. Knockdown of Evi blocks the export of Wnt 5a via MV in the same manner as via Exo indicating that it is active in both fractions and may be essential for the export of canonical as well as non-canonical Wnts in general.

We took particular care to rule out insufficient purity and mutual contamination of the particle preparations by using an elaborate centrifugation protocol followed by an additional filtration step to separate MV from the smaller Exo. The MV preparations did not contain particles $<100$ $\mathrm{nm}$, however, an overlap in the range of $100-200 \mathrm{~nm}$ cannot be completely excluded. Recently, it has become a matter of debate whether Exo and MV biogenesis can be strictly distinguished. Exosomal and endosomal proteins have been found enriched in certain regions of the plasma membrane which can serve as sites of direct Exo generation [32]. However, we identified a different pattern of protein expression on Exo and MV. The plasma membrane-bound EMMPRIN could be established as a specific marker of MV while TSG101, implicated in the biogenesis of MVB, was present predominantly on Exo. Equally, CD63 expression was restricted to Exo. This strongly suggests that we indeed obtained two populations with different biological characteristics and that Evi plays a role in both.

These extracellular particles are critical mediators of $M \Phi$-induced invasion as proven by the fact that the respective particle-free supernatant had no effect, although it should still contain all of the soluble factors we have identified earlier as indispensable for invasion [33]. Obviously, MФ-derived MV and Exo serve as carriers of Wnt 5a to the tumor cells where, after uptake, it becomes detectable intracellularly. Wnt $5 \mathrm{a}$ on these particles is biologically active and essential for induction of tumor cell invasion since Wnt 5a-negative P-MV do not enhance invasion, and Wnt 5a depletion via Evi knockdown antagonizes the proinvasive effect. The fact that invasion induced by Wnt 5a-positive MV from SK-BR-3 cells is only partially reversed by Wnt 5 a depletion implicates that there are additional vesicle-associated factors involved.

The way how T-MV and T-Exo, in contrast to vesicles of benign provenience, selectively induce Wnt 5a in $\mathrm{M} \Phi$ and whether they act at the cell membrane or intracellularly is still not completely clear. MV are known to be non-specifically ingested via endocytosis [34]. Consistently, MФ rapidly incorporated T- as well as P-MV. However, MV uptake alone did not decide on Wnt 5a induction since this was caused only by T-MV. Moreover, inhibition of endocytosis by dynasore did not counteract Wnt 5a upregulation and subsequent export. This argues against a similar effect as recently described for fibroblastinduced cancer cell motility [35]. There, fibroblast-derived Exo were internalized into breast cancer cells, loaded with cancer cell-derived Wnt 11 and then recycled to activate non-canonical Wnt signaling in these cells. Our findings, in contrast, suggest a still to be defined ligand-receptor interaction at the $\mathrm{M} \Phi$ surface.

Members of the Wnt family are potential candidates for this interaction since we demonstrate here that the Wnt inhibitor DKK-1 antagonizes vesicle-triggered Wnt 5a induction. Previously, we reported that DKK-1 counteracts tumor cell invasion induced by coculture with whole $М \Phi$. However, it remained unclear how DKK-1, considered predominantly an antagonist of $\beta$-Catenin-dependent Wnt signaling, could inhibit MФ-induced invasion which was mediated by non-canonical Wnt signaling via activation of JNK in the tumor cells [6]. Our present data suggest that DKK-1 exerts its main effect not on the tumor cells but on $М \Phi$ where it blocks the shift to a Wnt 5a-positive proinvasive phenotype as well as the production of Wnt 5a-carrying particles. Consistently, it does not antagonize invasion induced by Wnt 5a-positive MФ-MV from prestimulated MФ. Our observation that exposure of MФ to T-MV leads to p38/MAPK phosphorylation which has been implicated in $\beta$-Catenin-dependent Wnt signaling [36] may further support this assumption. P38 phosphorylation is effectively counteracted by DKK-1. Inhibition of p38 phosphorylation by a specific antagonist, in turn, downregulates Wnt 5a induction.

In conclusion, extracellular vesicles and not soluble factors as expected are the essential communicators between $M \Phi$ and cancer cells during $M \Phi$-induced malignant invasion. Surprisingly, both populations, although of different origin, exert a similar biological function. As shown in figure 8, they partake in a reciprocal loop where malignant $\mathrm{MV}$ and Exo induce production of Wnt $5 \mathrm{a}$ in $\mathrm{M \Phi}$, a step which is sensitive to Wnt antagonists. MФ-derived Wnt $5 \mathrm{a}$ is then delivered via responding $M \Phi-M V$ and Exo to the recipient cells and enhances invasion. Transport of Wnt 5a in both populations may occur partly in a non-specific way but is also dependent on the presence of Evi. Thus, Evi seems involved in the secretion of canonical as well as noncanonical Wnts and exerts its cargo function in a more general fashion on extracellular vesicles with different biological characteristics. 


\section{MATERIAL AND METHODS}

\section{Cells, viability and lentiviral production}

The human breast cancer cell lines MCF-7 and SKBR-3 (ATCC) were grown in RPMI-1640 (PAA) + 10\% fetal calf serum (FCS). Murine L cells overexpressing Wnt5a (Wnt 5a-L cells) were maintained in DMEM (Biochrom) $+10 \%$ FCS. Human M $\Phi$ were derived from peripheral blood mononuclear cells as described [6] and cultured in RPMI-1640 + 1\% FCS. Viability upon treatment with ActD $(32 \mu \mathrm{M}$, Lundbeck Pharmaceuticals Ireland Limited), DKK-1 (200 ng/ml, R\&D systems) and SB-203580 (0,5 $\mu \mathrm{M}$, Calbiochem) was tested with the MTT assay using standard protocols. For generation of SK-BR-3 and Wnt5a-L cells with stable shEvi expresssion HEK293T cells were cotransfected with the packaging plasmids pVSG-G and pCMV $\triangle$ R8.91 and the pLKO shRNA control or shEvi plasmid (Sigma) through calcium phosphate precipitation. The ns ctl sequence is 5'-CCCGTGTAAATATGTACATTT-3', the Evi targeting sequence is $5^{\prime}$-GATCTACAAGTTGACCCGCAA-3'. The virus-containing supernatant was concentrated using lentiviral enrichment reagent (MobiTech). Cells were selected in medium with $2 \mu \mathrm{g} / \mathrm{mL}$ (SK-BR-3) or $15 \mu \mathrm{g} / \mathrm{ml}$ ( $\mathrm{L}$ cells) puromycin (Sigma).

\section{Isolation of MV and Exo, and calcein staining}

Cells were cultured up to $48 \mathrm{~h}$ in medium + heatinactivated FCS (particle-free through ultracentrifugation, $100.000 \mathrm{~g}$, overnight). Supernatants were centrifuged at $750 \mathrm{~g}(5 \mathrm{~min})$ and $1,500 \mathrm{~g}(15 \mathrm{~min})$ to remove cells and debris, followed by ultracentrifugation $(14,000 \mathrm{~g}, 35 \mathrm{~min}$, $4^{\circ} \mathrm{C}$ ) to precipitate MV. Pellets were resuspended in $200 \mu \mathrm{l}$ PBS or RIPA lysis buffer for protein quantification $\left(D_{c}\right.$ protein assay, Bio-Rad). MV membrane integrity was determined by staining with calcein AM (MobiTec). The signal was detected by flow cytometry and related to the acetone-treated negative controls. P-MV were isolated from expired ( $<2$ days) platelet concentrates from healthy blood donors following the same protocol. For Exo preparations, the supernatant after MV precipitation was filtered $(0,22 \mu \mathrm{m}$, Sarstedt) and ultracentrifuged at $100.000 \mathrm{~g}\left(4^{\circ} \mathrm{C}, 2 \mathrm{~h}\right)$. The Exo pellet was washed once in PBS before protein quantification.

\section{Flow cytometry}

Exosomes were coupled to latex beads (aldehyde sulfate, $4 \mu \mathrm{m}$, Invitrogen) as described previously [37]. Briefly, exosomes and beads were incubated in MES buffer $(0,025 \mathrm{M}$ MES, $0,154 \mathrm{M} \mathrm{NaCl}, \mathrm{pH} 6)$ for $1 \mathrm{~h}$ at room temperature before incubating overnight at $4{ }^{\circ} \mathrm{C}$ with gentle shaking. Beads were blocked with $200 \mathrm{mM}$ glycine for $30 \mathrm{~min}$ and washed twice in PBS + 1\% FCS (particlefree). MV and exosomes were stained with primary antibodies against EMMPRIN (\#sc-13976, Santa Cruz) or CD63 (\#556019, BD), washed twice in PBS and incubated with FITC-labeled anti-rabbit (\#406403, BioLegends) or anti-mouse (\#sc-2010, Santa Cruz) secondary antibodies. Signals were detected with a FACS Canto II flow cytometer. MV and exosome populations were gated and analyzed with the FACS Diva (version 6.1.3.) software.

\section{Quantitative real-Time RT-PCR}

$\mathrm{M} \Phi$ and MCF-7 cells were stimulated for $24 \mathrm{~h}$ with $100 \mathrm{ng} / \mathrm{ml}$ LPS (Sigma-Aldrich) or MV/Exo at the indicated concentrations. Total RNA was extracted with the High Pure RNA Isolation kit (Roche) and reverse transcription was performed from $1 \mu \mathrm{g}$ of total RNA with the iscript cDNA synthesis kit (Bio-Rad). The cDNA products were subjected to quantitative RT-PCR with SYBR green detection (7900 HT system, Applied Biosystems). The primer sequences are listed in suppl. Tab. 1 or for WNT5A published in [8].

\section{Western Blot, ELISA and sucrose gradient}

Cells were lysed and homogenized in RIPA lysis buffer $(150 \mathrm{mM} \mathrm{NaCl} / 0,1 \% \mathrm{SDS} / 0,5 \%$ Na-deoxycholate/ $1 \%$ Triton X-100/ $50 \mathrm{mM}$ Tris, $\mathrm{pH} 7,2)$. Up to $50 \mu \mathrm{g}$ of total protein were subjected to SDS-PAGE $(8 \%)$ and blotted onto a nitrocellulose membrane (Amersham Biosciences). Ponceau S staining was used as loading control. Membranes were incubated with antibodies specific to EMMPRIN (\#sc-13976, Santa Cruz), Wnt5a (\#MAB645, R\&D systems), TSG101 (\#7964, Santa Cruz), EVI (\#MABS87, Millipore), p38 MAPK total protein (\#9212) and phospho-p38 MAPK (\#9211), both from Cell Signaling. Signals were detected with ECL ${ }^{\mathrm{TM}}$ Prime (Amersham Biosciences). Cytokine concentrations in the supernatants were analyzed with Legend $\mathrm{Max}^{\mathrm{TM}}$ ELISA kits (BioLegend, IL-1ß \#437007, IL-12 p70 \#431707, IL10 \#430607) according to the manufacturer's instructions. LPS (100 ng/ml, E. coli K-235, Sigma) was used as a positive control. For sucrose gradient preparations, SKBR-3 supernatant was centrifuged at $750 \mathrm{~g}$ and $1500 \mathrm{~g}$ as described above and directly applied to ultracentrifugation (see isolation of MV and Exo). The pellet was washed in PBS and layered on top of a sucrose step gradient $(0,25$ $2,25 \mathrm{M})$ which was ultracentrifuged for $16 \mathrm{~h}$ at $100.000 \mathrm{~g}$. Eight fractions $(2 \mathrm{ml})$ were collected and precipitated with ice-cold acetone $(1: 10, \mathrm{v} / \mathrm{v})$ overnight at $-20^{\circ} \mathrm{C}$. 


\section{Electron microscopy}

MV and Exo pellets were resuspended in $0,1 \mathrm{M}$ sodium phosphate $+2 \%$ paraformaldehyde, applied to carbon-coated EM grids (400 mesh, Plano GmbH) and postfixed in $1 \%$ glutaraldehyde. The grid was washed with $\mathrm{dH}_{2} \mathrm{O}$ and incubated in $1 \%$ uranyl acetate $(30 \mathrm{~s})$ for negative contrasting. MV were visualized using the $\mathrm{CM}$ 120 Bio Twin transmission electron microscope (Philips) and iTEM software (Olympus). For detection of Wnt5a, MV pellets were stained with above named Wnt5a antibody and an immunogold-labeled secondary antibody (\#810.077, Aurion).

\section{Microinvasion assay and MV uptake}

Invasion was quantified using a modified Boyden chamber assay as described [32]. For uptake, tumor cells were exposed to MV/Exo in the indicated concentrations and the according particle-free supernatants. MV uptake was measured using MV stained with the red-fluorescent membrane dye PKH26 (Sigma-Aldrich) according to the manufacturer's instructions. Staining efficiency was tested by flow cytometry. Cells were incubated with the labeled MV $(5 \mu \mathrm{g} / \mathrm{ml}, 24 \mathrm{~h})$ and analyzed by confocal/fluorescence microscopy (Axiovert $200 \mathrm{M}$ and LSM 510 Meta, Zeiss). For quantification, the PKH26 fluorescence in cells and supernatant was measured by flow cytometry.

\section{Statistical analysis}

Graphs were created with GraphPad Prism for Windows (version 5.04, GraphPad software). Data are means $\pm \mathrm{SD}$ and were compared with the twotailed Student's t-test, $\mathrm{p}$-values $<0,05$ were considered significant.

\section{ACKNOWLEDGEMENTS}

This work was supported by grants nr. 109615 (Deutsche Krebshilfe) and BI 703/3-2 (DFG). We thank Lena Ries, Meike Schaffrinski and Matthias Schulz for technical assistance.

\section{REFERENCES}

1. Qian BZ and Pollard JW. Macrophage Diversity Enhances Tumor Progression and Metastasis. Cell. 2010; 141(1):3951.

2. Sica A, Schioppa T, Mantovani A and Allavena P. Tumourassociated macrophages are a distinct M2 polarised population promoting tumour progression: Potential targets of anti-cancer therapy. Eur J Cancer. 2006; 42(6):717-727.

3. Balkwill F. Tumour necrosis factor and cancer. Nat Rev
Cancer. 2009; 9(5):361-371.

4. Kessenbrock K, Plaks V and Werb Z. Matrix Metalloproteinases: Regulators of the Tumor Microenvironment. Cell. 2010; 141(1):52-67.

5. Wyckoff J, Wang WG, Lin EY, Wang YR, Pixley F, Stanley ER, Graf T, Pollard JW, Segall J and Condeelis J. A paracrine loop between tumor cells and macrophages is required for tumor cell migration in mammary tumors. Cancer Res. 2004; 64(19):7022-7029.

6. Pukrop T, Klemm F, Hagemann T, Gradl D, Schulz M, Siemes S, Trumper L and Binder C. Wnt 5a signaling is critical for macrophage-induced invasion of breast cancer cell lines. P Natl Acad Sci USA. 2006; 103(14):5454-5459.

7. Clevers H and Nusse R. Wnt/beta-Catenin Signaling and Disease. Cell. 2012; 149(6):1192-1205.

8. Weeraratna AT, Jiang YA, Hostetter G, Rosenblatt K, Duray P, Bittner M and Trent JM. Wnt5a signaling directly affects cell motility and invasion of metastatic melanoma. Cancer Cell. 2002; 1(3):279-288.

9. Jiang W, Crossman DK, Mitchell EH, Sohn P, Crowley MR and Serra R. WNT5A Inhibits Metastasis and Alters Splicing of Cd44 in Breast Cancer Cells. Plos One. 2013; $8(3)$.

10. Blumenthal A, Ehlers S, Lauber J, Buer J, Lange C, Goldmann T, Heine H, Brandt E and Reiling N. The Wingless homolog, WNT5A and its receptor Frizzled-5 regulate inflammatory responses of human mononuclear cells induced by microbial stimulation. Blood. 2006; 108(3):965-973.

11. Bartscherer K and Boutros M. Regulation of Wnt protein secretion and its role in gradient formation. Embo Rep. 2008; 9(10):977-982.

12. Port F and Basler K. Wnt Trafficking: New Insights into Wnt Maturation, Secretion and Spreading. Traffic. 2010; 11(10):1265-1271.

13. Thery C, Ostrowski M and Segura E. Membrane vesicles as conveyors of immune responses. Nat Rev Immunol. 2009; 9(8):581-593.

14. Gyorgy B, Szabo TG, Pasztoi M, Pal Z, Misjak P, Aradi B, Laszlo V, Pallinger E, Pap E, Kittel A, Nagy G, Falus A and Buzas EI. Membrane vesicles, current state-of-the-art: emerging role of extracellular vesicles. Cell Mol Life Sci. 2011; 68(16):2667-2688

15. Thaler J, Ay C, Weinstabl H, Dunkler D, Simanek R, Vormittag R, Freyssinet JM, Zielinski C and Pabinger I. Circulating procoagulant microparticles in cancer patients. Ann Hematol. 2011; 90(4):447-453.

16. Muralidharan-Chari V, Clancy JW, Sedgwick A and D'Souza-Schorey C. Microvesicles: mediators of extracellular communication during cancer progression. J Cell Sci. 2010; 123(10):1603-1611

17. Bianco F, Perrotta C, Novellino L, Francolini M, Riganti L, Menna E, Saglietti L, Schuchman EH, Furlan R, Clementi E, Matteoli M and Verderio C. Acid sphingomyelinase 
activity triggers microparticle release from glial cells. Embo J. 2009; 28(8):1043-1054.

18. Gross JC, Chaudhary V, Bartscherer K and Boutros M. Active Wnt proteins are secreted on exosomes. Nat Cell Biol. 2012; 14(10):1036-+.

19. Korkut C, Ataman B, Ramachandran P, Ashley J, Barria $\mathrm{R}$, Gherbesi N and Budnik V. Trans-Synaptic Transmission of Vesicular Wnt Signals through Evi/Wntless. Cell. 2009; 139(2):393-404.

20. Azmi AS, Bao B and Sarkar FH. Exosomes in cancer development, metastasis, and drug resistance: a comprehensive review. Cancer metastasis reviews. 2013.

21. Abrahams VM, Straszewski SL, Kamsteeg M, Hanczaruk B, Schwartz PE, Rutherford TJ and Mor G. Epithelial ovarian cancer cells secrete functional Fas ligand. Cancer Res. 2003; 63(17):5573-5581.

22. Baran J, Baj-Krzyworzeka M, Weglarczyk K, Szatanek R, Zembala M, Barbasz J, Czupryna A, Szczepanik A and Zembala M. Circulating tumour-derived microvesicles in plasma of gastric cancer patients. Cancer Immunol Immun. 2010; 59(6):841-850.

23. Al-Nedawi K, Meehan B, Micallef J, Lhotak V, May L, Guha A and Rak J. Intercellular transfer of the oncogenic receptor EGFrvIII by microvesicles derived from tumour cells. Nat Cell Biol. 2008; 10(5):619-U624.

24. Obregon C, Rothen-Rutishauser B, Gitahi SK, Gehr P and Nicod LP. Exovesicles from human activated dendritic cells fuse with resting dendritic cells, allowing them to present alloantigens. Am J Pathol. 2006; 169(6):2127-2136.

25. Frey B and Gaipl US. The immune functions of phosphatidylserine in membranes of dying cells and microvesicles. Semin Immunopathol. 2011; 33(5):497-516.

26. Vega VL, Rodriguez-Silva M, Frey T, Gehrmann M, Diaz JC, Steinem C, Multhoff G, Arispe N and De Maio A. Hsp70 translocates into the plasma membrane after stress and is released into the extracellular environment in a membrane-associated form that activates macrophages. J Immunol. 2008; 180(6):4299-4307.

27. Koppler B, Cohen C, Schlondorff D and Mack M. Differential mechanisms of microparticle transfer to B cells and monocytes: anti-inflammatory properties of microparticles. Eur J Immunol. 2006; 36(3):648-660.

28. Baj-Krzyworzeka M, Szatanek R, Weglarczyk K, Baran J, Urbanowicz B, Branski P, Ratajczak MZ and Zembala M. Tumour-derived microvesicles carry several surface determinants and mRNA of tumour cells and transfer some of these determinants to monocytes. Cancer Immunol Immun. 2006; 55(7):808-818.

29. Grange C, Tapparo M, Collino F, Vitillo L, Damasco C, Deregibus MC, Tetta C, Bussolati B and Camussi G. Microvesicles Released from Human Renal Cancer Stem Cells Stimulate Angiogenesis and Formation of Lung Premetastatic Niche. Cancer Res. 2011; 71(15):5346-5356.

30. Castellana D, Zobairi F, Martinez MC, Panaro MA,
Mitolo V, Freyssinet JM and Kunzelmann C. Membrane Microvesicles as Actors in the Establishment of a Favorable Prostatic Tumoral Niche: A Role for Activated Fibroblasts and CX3CL1-CX3CR1 Axis. Cancer Res. 2009; 69(3):785793.

31. Dashevsky O, Varon D and Brill A. Platelet-derived microparticles promote invasiveness of prostate cancer cells via upregulation of MMP-2 production. Int J Cancer. 2009; 124(8):1773-1777.

32. Booth AM, Fang Y, Fallon JK, Yang JM, Hildreth JEK and Gould SJ. Exosomes and HIV Gag bud from endosome-like domains of the T cell plasma membrane. J Cell Biol. 2006; 172(6):923-935.

33. Hagemann $\mathrm{T}$, Robinson SC, Schulz M, Trumper L, Balkwill FR and Binder C. Enhanced invasiveness of breast cancer cell lines upon co-cultivation with macrophages is due to TNF-alpha dependent up-regulation of matrix metalloproteases. Carcinogenesis. 2004; 25(8):1543-1549.

34. Kawamoto T, Ohga N, Akiyama K, Hirata N, Kitahara S, Maishi N, Osawa T, Yamamoto K, Kondoh M, Shindoh M, Hida Y and Hida K. Tumor-Derived Microvesicles Induce Proangiogenic Phenotype in Endothelial Cells via Endocytosis. Plos One. 2012; 7(3).

35. Luga V, Zhang L, Viloria-Petit AM, Ogunjimi AA, Inanlou MR, Chiu E, Buchanan M, Hosein AN, Basik M and Wrana JL. Exosomes Mediate Stromal Mobilization of Autocrine Wnt-PCP Signaling in Breast Cancer Cell Migration. Cell. 2012; 151(7):1542-1556.

36. Cervenka I, Wolf J, Masek J, Krejci P, Wilcox WR, Kozubik A, Schulte G, Gutkind JS and Bryja V. MitogenActivated Protein Kinases Promote WNT/beta-Catenin Signaling via Phosphorylation of LRP6. Mol Cell Biol. 2011; 31(1):179-189.

37. Lässer C, Eldh, M., Lötvall, J. Isolation and Characterization of RNA-Containing Exosomes. J Vis Exp. 2012; 9(59):e3037 3010.3791/3037. 